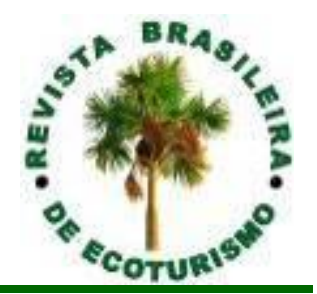

\title{
Percepção dos serviços ambientais gerados por uma área natural de alto valor turístico: Cachoeira do Roncadeira (TO)
}

\author{
Perception about ecosystem services provided by a natural area \\ with high touristic value: Roncadeira Waterfall (TO, Brazil)
}

\author{
Nibelle Aires Lira, Fernando Mayer Pelicice
}

RESUMO: Os ecossistemas fornecem benefícios fundamentais às sociedades humanas, conhecidos como serviços ambientais (ou ecossistêmicos). O desconhecimento sobre esses serviços alimenta a crença de autonomia das atividades humanas, precipitando a perda de ecossistemas, biodiversidade e degradação ambiental. Nesse contexto, o presente trabalho investigou como as pessoas percebem a importância e os benefícios dos serviços ecossistêmicos prestados por uma área de elevado potencial turístico e recreativo, no caso, a Cachoeira do Roncadeira, localizada no distrito de Taquaruçu, Palmas (Tocantins). O estudo entrevistou pessoas na área da cachoeira (grupo Cachoeira) e em centros urbanos da cidade de Palmas (grupo Urbano), entre janeiro e fevereiro de 2013. Utilizando questionário estruturado, avaliou-se (i) o perfil do entrevistado, (ii) a frequência de uso da cachoeira, e (iii) a relação entre usuário e ecossistema. Com relação ao último ponto, investigamos a compreensão do entrevistado em relação à importância de 11 serviços ecossistêmicos gerados pela cachoeira e seu entorno, sua percepção quanto aos benefícios gerados pelos serviços, e 0 seu posicionamento quanto à perda ou transformação da área natural. A pesquisa entrevistou 45 pessoas no grupo Urbano e 109 no grupo Cachoeira. No geral, o perfil das respostas foi muito semelhante entre os dois grupos. A maior parte dos entrevistados declarou frequentar áreas naturais entre 1 e 3 vezes por ano. De maneira geral, as pessoas entrevistadas apresentaram compreensão heterogênea sobre a importância dos serviços ambientais. Alguns serviços foram bem reconhecidos (i.e. biodiversidade, recursos hídricos, valor cultural, educação e regulação do clima), porém outros foram mal valorizados (i.e. produção de alimentos, matéria-prima, combustível e controle de doenças). Quase todos os entrevistados declararam sentir-se beneficiados por algum serviço, especialmente aqueles atrelados ao turismo (i.e. biodiversidade, recreação e cultura); no entanto, o percentual de beneficiados (declarados) foi baixo para a maior parte dos serviços. Aproximadamente metade dos entrevistados se posicionou contra qualquer tipo de modificação na área; os demais demonstraram interesse na implantação de alguma atividade econômica na região. Os resultados indicam, de maneira geral, que as pessoas têm compreensão limitada sobre a importância do ecossistema ripário como provedor de serviços ambientais, apontando a necessidade de esclarecimento, para o público geral e autoridades, sobre o funcionamento dos sistemas naturais, seus serviços e sua relação com o bem-estar humano. O ecoturismo pode desempenhar papel positivo nesse processo.

PALAVRAS CHAVE: Áreas Ripárias; Bem-Estar; Economia; Sustentabilidade; Serviços Ecossistêmicos. 
ABSTRACT: Ecosystems provide fundamental benefits to human societies, known as ecosystem services. Ignorance about these services supports the notion that human activities are autonomous, driving the loss of ecosystems, biodiversity and environmental degradation. In this context, the present study investigated how people perceive the importance and benefits of ecosystem services provided by a natural area of high touristic value, in the case, the Roncadeira Waterfall, located in Taquaruçu, Palmas (Tocantins, Brazil). We interviewed people in the area of the waterfall (Waterfall group) and in urban centers in Palmas (Urban group), between January and February 2013. Using a structured questionnaire, we assessed the (i) interviewee's profile, (ii) the frequency use of the waterfall, and (iii) the relationship between people and the ecosystem. Regarding the last issue, we investigated how interviewees perceive the importance of 11 ecosystem services generated by the waterfall and its region, the benefits generated by these services, and how people react to the loss or transformation of the natural area. The research interviewed 45 people in the Urban group and 109 in the Waterfall group. In general, responses were similar between groups. Most interviewees stated that they visit natural areas between 1 and 3 times a year. In general, they showed a heterogeneous perception about the importance of ecosystem services. Some services were well recognized (i.e. biodiversity, water resources, cultural value, education and climate regulation), but others were poorly valued (i.e. food production, raw material, fuel and disease control). Almost all respondents felt benefited by some service, especially those linked to tourism (i.e. biodiversity, recreation and culture); however, the percentage of benefited people (declared) was low for most services. Approximately half of the interviewees stood against any kind of modification in the area; the remaining showed interest in the implementation of some economic activity. Concluding, our results indicated that people have limited understanding about the importance of the riparian environment as a source of ecosystem services, pointing out the need for clarification of the public and authorities about the functioning of natural systems, their services and their relation with human well-being. Ecotourism can play a positive role in this process.

KEYWORDS: Riparian Areas; Well-Being; Economy; Sustainability; Ecosystem Services.

\section{Introdução}

Os ecossistemas estão na base das atividades humanas, seja pela provisão de recursos, seja pela regulação de processos no planeta Terra. A sociedade humana obtém dos ecossistemas uma gama variada de benefícios diretos e indiretos, conhecidos como serviços ecossistêmicos (ou ambientais), fundamentais à manutenção dos sistemas urbanos, agrários e indústria (DAILY et al., 1997; TURNER et al., 2007; BURGER et al., 2015). Dentre serviços essenciais e insubstituíveis estão, por exemplo, a produção de água nos mananciais, purificação do ar, ciclagem de nutrientes, polinização, produção de alimentos (naturais e cultivados), controle de doenças, proteção contra catástrofes naturais, regulação climática, fatores estéticos e estímulo intelectual (MILLENIUM ECOSYSTEM ASSESSMENT, 2005a; COSTANZA et al., 2017). Em linhas gerais, os ecossistemas naturais produzem serviços de provisão, regulação, suporte e de valor cultural, os quais se originam de complexas relações entre processos naturais de ordem física, química e biológica, tendo a biodiversidade um papel preponderante (NAEEM et al., 1999; HOOPER et al., 2005; CADOTTE et al., 2011).

No entanto, a expansão das atividades humanas, para atender a crescentes demandas por alimentos, água, matéria prima e energia, têm causado alterações sem precedentes nos padrões de biodiversidade e no funcionamento dos 
ecossistemas naturais (VITOUSEK et al., 1997; CEBALLOS et al., 2015). O declínio das áreas naturais afeta atributos de resiliência e persistência dos ecossistemas, alterando ou diminuindo sua capacidade em manter processos naturais e, portanto, prover serviços fundamentais para as atividades humanas (VOROSMARTY et al., 2010; NAIMAN; DUDGEON, 2011; CARDINALE et al., 2012). A desinformação do público em geral e dos tomadores de decisão sobre o funcionamento dos sistemas naturais é fator preponderante precipitando a perda de ecossistemas e biodiversidade; inclusive, estimula a percepção de independência da sociedade humana, resultando na desvalorização dos serviços prestados. Isso realça a necessidade de se compreender a maneira como a sociedade percebe e julga a existência e relevância dos serviços ecossistêmicos. Investigar essa relação é importante, pois práticas mais sustentáveis, e um melhor alinhamento entre produção e manutenção de sistemas naturais, dependerá de mudanças comportamentais. Uma sociedade mais esclarecida deve naturalmente valorizar o sistema no qual ela depende.

No geral, a compreensão das pessoas em relação aos benefícios fornecidos pelos sistemas naturais está usualmente limitada aos serviços de provisão (alimentos, combustível, fármacos) e culturais (turismo e lazer). Essa percepção é fomentada pelo contato direto que as pessoas têm com o recurso natural e seus benefícios. Um caso típico é o uso de áreas naturais ripárias, ambientes formados por corpos d'água (lagos, rios) e a vegetação circundante (mata ciliar ou de galeria), sendo muito apreciadas para fins turísticos e de recreação. Praias fluviais, lagoas e cachoeiras, por exemplo, são ambientes muito visitados em todo o Brasil, e adquirem valor turístico na medida em que suas condições naturais são preservadas. Entretanto, a importância desses ambientes vai muito além do turismo, visto que desempenham serviços essenciais, tais como a proteção do manancial, produção de água potável, controle de erosão das margens, ciclagem e retenção de nutrientes e sedimentos (MCCLAIN; COSSIO, 2003; VOROSMARTY et al., 2010; BIGGS et al., 2017). É muito provável, entretanto, que tais serviços permaneçam desconhecidos ou mal dimensionados pelas pessoas no geral, incluindo usuários que se beneficiam diretamente desses ambientes (i.e. turistas). Dessa maneira, é importante conhecer a percepção das pessoas em relação aos serviços e funções ecossistêmicas prestados por ambientes ripários, a fim de estabelecer ações de educação ambiental que busquem a mudança de comportamento, promovendo atitudes orientadas à conservação dessas áreas. Seu potencial turístico, inclusive, pode catalisar o processo de aprendizado, já que existe empatia das pessoas pelo ambiente, contexto em que o ecoturismo pode ser ferramenta importante.

Nesse cenário, o presente trabalho teve como objetivo compreender a maneira com que as pessoas percebem a importância e os benefícios dos serviços ecossistêmicos prestados por uma área de elevado potencial turístico e recreativo, no caso, a Cachoeira do Roncadeira, localizada no distrito de Taquaruçu, Palmas, Estado do Tocantins. Para isso, o estudo entrevistou moradores da cidade de Palmas e frequentadores da referida cachoeira, a fim de investigar o uso da área e a relação entre as pessoas e o ambiente. Espera-se que os resultados encontrados neste trabalho ajudem a direcionar programas de educação ambiental específicos, incluindo o ecoturismo, no sentido de esclarecer questões relacionadas ao papel dos sistemas naturais e sua relação com o bem-estar humano. 


\section{Material e Métodos}

\section{Caracterização da área de estudo}

Taquaruçu é um distrito que se localiza na parte centro sul do município de Palmas, capital do Estado do Tocantins. O distrito apresenta área total de 63.918,45 ha e localiza-se dentro de uma APA (área de proteção ambiental), criada com vista à conservação dos mananciais que abastecem parte da população de Palmas. $\mathrm{O}$ distrito tem elevado apelo turístico, com a presença de muitas cachoeiras, trilhas e eventos culturais, alguns de reputação nacional (e.g. Festival Gastronômico de Taquaruçu).

A Cachoeira do Córrego Roncadeira (10¹8'13.26"S 4808'20.39"O) está inserida em uma propriedade particular (Fazenda Dom Manuel), localizada na rodovia TO-030, saída norte do distrito. Possui queda d'água de aproximadamente 70 m, e faz parte do Ribeirão Taquaruçu, que deságua no rio Tocantins (reservatório de Lajeado). Em virtude de sua localização e fácil acesso (trilha ecológica de 1500 $\mathrm{m})$, a propriedade recebe elevado fluxo de visitação de turistas, principalmente durante feriados e finais de semana. Essa cachoeira está entre as principais do distrito, tendo como principal atrativo sua elevada queda d'água e trilhas (Figura 1). Possui sede administrativa que regula a entrada e permanência dos visitantes.
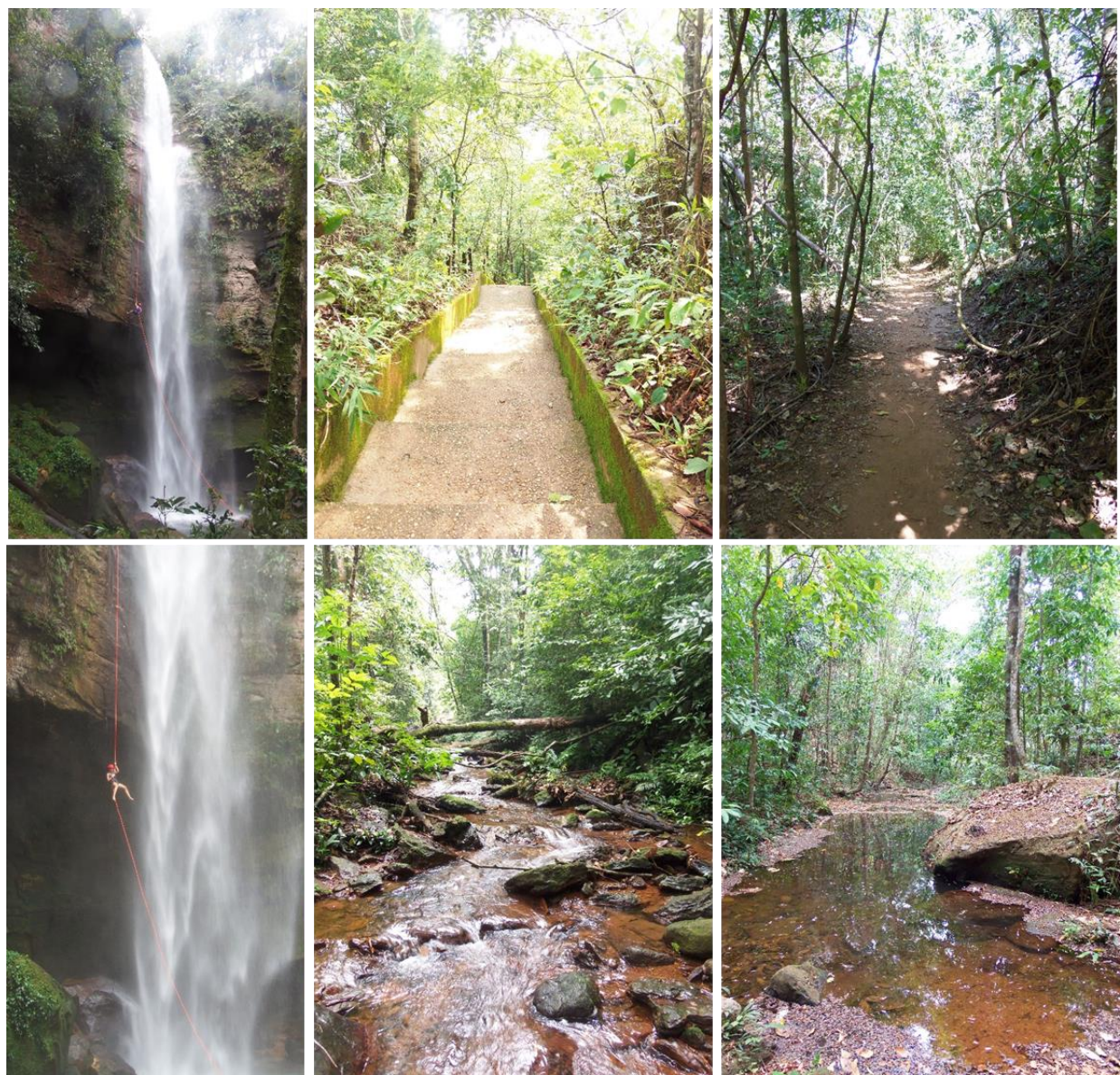

Figura 1: Imagens da área de estudo, contemplando a cachoeira do Roncadeira, a trilha ecológica e o córrego, distrito de Taquaruçu, Palmas, Tocantins. Fonte: elaborado pelos autores (2020).

Figure 1: Scenes from the study area, depicting the Roncadeira Waterfall, the ecological trail and the stream, Taquaruçu, Palmas, Tocantins. Source: elaborated by the authors (2020). 


\section{Coleta dos dados}

Foram aplicados questionários estruturados, respondidos individualmente entre janeiro e fevereiro de 2013. Os questionários foram aplicados em dois cenários, que formaram dois grupos de respondentes: (i) grupo Cachoeira, composto por pessoas entrevistadas na própria Cachoeira da Roncadeira; (ii) grupo Urbano, composto por pessoas entrevistadas em centros urbanos da cidade de Palmas (Shopping Capim Dourado, Palmas Shopping e Feira 304 Sul). Todos as pessoas responderam ao questionário voluntariamente e por livre consentimento, após solicitação e esclarecimento do pesquisador. $\mathrm{Na}$ abordagem inicial, o pesquisador convidava à participação, explicava sinteticamente os objetivos do projeto, assegurava o anonimato do respondente, e esclarecia o uso dos dados gerados. A aplicação do questionário foi conduzida pelo pesquisador.

O questionário contemplou três domínios de informação: A. Perfil do entrevistado; B. Uso de áreas naturais; C. Relação indivíduo x ambiente (Tabela 1).

Tabela 1. Questionário estruturado aplicado na entrevista das pessoas. Table 1. Structured questionnaire used in the interviews.

\section{VARIÁVEIS}

\section{A. PERFIL}

1. Sexo: ( ) Masculino ( ) Feminino

2. Residência (cidade):

3. Idade:

4. Escolaridade: ( ) Fundamental incompleto ou ( ) completo; ( ) Médio incompleto ou ( ) completo; ( ) Superior incompleto ou ( ) completo; ( ) Pós-graduação incompleta ou ( ) completa

\section{B. USO DE ÁREAS NATURAIS}

5. Quantas vezes você visita esse local (por ano)?

( ) 1 vez; ( ) 2 vezes; ( ) 5 vezes; ( ) 5-10; ( ) > 10

6. Quantas vezes você costuma visitar áreas naturais como essa?

( ) 1 vez; ( ) 2 vezes; ( ) 5 vezes; ( ) 5-10; ( ) > 10

\section{RELAÇÃO COM O AMBIENTE}

7. Como você percebe a importância desta área para cada aspecto:

( ) nula ( ) fraca ( ) média ( ) forte

(i) Biodiversidade, (ii) Recreação, (iii) Recursos hídricos, (iv) Purificação de água, (v)

Produção de alimentos, (vi) Produção de matéria-prima, (vii) Produção de combustível, (viii)

Valor cultural, (ix) Educação, (x) Controle de doenças, (xi) Regulação do clima

8. Você se vê beneficiado com algum dos serviços citados acima?

( ) Sim. Quais:

( ) Não

9. Se houvesse uma iniciativa em transformar essa área num sistema de produção econômica, para gerar renda, emprego e capital, qual das atividades você acharia mais interessante

( ) Pecuária; ( ) Agricultura; ( ) Hidrelétrica; ( ) Parque industrial; ( ) Mineração; ( ) Urbanização; ( ) Nenhuma.

10. Se houvesse um projeto para desenvolver economicamente o Estado do Tocantins, que resultasse na perda de diversas áreas como essa, você concordaria?

( ) Sim; ( ) Não; ( ) Somente se o ganho econômico for muito elevado 
As perguntas do domínio $\mathrm{A}$ foram elaboradas com a finalidade de caracterizar o perfil do entrevistado. O domínio B teve como foco investigar a frequência de visitação à Cachoeira do Roncadeira e outras áreas naturais. As questões do domínio $\mathrm{C}$ foram delineadas para investigar a compreensão do entrevistado em relação à relevância dos serviços ecossistêmicos oferecidos pela cachoeira e seu entorno. Para isso, elencamos onze serviços ecossistêmicos prestados por zonas ripárias reconhecidos na literatura (POSTEL; CARPENTER, 1997; DAILY et al., 1997; MILLENIUM ECOSYSTEM ASSESSMENT, 2005b; AUERBACH et al., 2014; BIGGS et al., 2017): Biodiversidade, Recreação, Abastecimento de Água, Purificação de Água, Produção de Alimentos, Produção de Matéria-Prima, Produção de Combustível, Valor Cultural, Educação, Controle de Doenças e Regulação do Clima. O entrevistado indicou a importância de cada serviço (em uma escala: Nula, Fraca, Média e Forte), e quais serviços geram benefícios para sua vida. No domínio $\mathrm{C}$, também avaliamos o posicionamento do entrevistado frente a diferentes cenários de desenvolvimento econômico na área da Cachoeira e no Estado do Tocantins. Para isso, perguntamos se o entrevistado achava interessante o desenvolvimento de uma série de atividades econômicas na região (Pecuária, Agricultura, Hidrelétrica, Parque Industrial, Mineração, Urbanização, Nenhuma); e se concordava com um modelo de desenvolvimento econômico que provocasse perda de áreas naturais no Estado do Tocantins.

\section{Análise de dados}

Todas as análises consideraram separadamente os dois grupos de entrevistados (Urbano e Cachoeira), de modo a comparar suas respostas.

O perfil dos entrevistados foi avaliado considerando a proporção entre os sexos, idade média (mín-máx), nível de escolaridade (\% de respondentes entre os níveis) e residência (\% de respondentes nas regiões do país). Para avaliar a intensidade de visitação da cachoeira da Roncadeira e outras áreas naturais, calculamos o percentual de respondentes em cada nível de frequência.

Para investigar a compreensão dos respondentes com relação à importância da área na provisão dos serviços ecossistêmicos, calculamos o percentual de respostas em cada um dos níveis de importância (Nulo a Forte), individualmente para cada serviço. O benefício percebido de cada serviço foi investigado calculando o percentual de indivíduos que se autodeclararam beneficiados por cada serviço. Também calculamos o número de beneficiados agrupando os serviços ecossistêmicos em 3 categorias, seguindo Millenium Ecosystem Assessment (2005a): (i) Provisão (biodiversidade, abastecimento de água, produção de alimentos, produção de matéria prima e produção de combustível); (ii) Cultural (valor cultural, educação e recreação); e (iii) Regulação (controle doenças, regulação do clima e purificação de água). Das questões relacionadas ao posicionamento do entrevistado frente aos cenários de desenvolvimento econômico, calculou-se o percentual de respondentes nas diferentes classes de respostas. 


\section{Resultados}

\section{Perfil dos entrevistados}

A pesquisa contou com 45 pessoas no grupo Urbano e 109 no grupo Cachoeira, perfazendo um universo amostral de 154 indivíduos (Tabela 2). Em ambos os grupos, o percentual de mulheres/homens foi proporcional. A idade variou entre 18 e 50 anos no Urbano, e entre 18 e 67 no grupo Cachoeira. A maioria dos entrevistados declarou possuir nível de escolaridade elevado; cerca de $70 \%$ das pessoas concluiu ou está concluindo um curso superior. Foi observado ainda que daqueles que concluíram, cerca $20 \%$ possuem pós-graduação. Todos os entrevistados no grupo Urbano residem no Estado do Tocantins. A residência dos entrevistados no grupo Cachoeira está dividida principalmente entre a região norte (80\%), representado aqui pelo Estado do Tocantins, e a sudeste (11\%). Outras regiões somam um percentual de $9 \%$.

Tabela 2: Perfil dos entrevistados nos grupos Urbano e Cachoeira. Códigos: $\mathrm{N}=$ número de entrevistados; $M=$ masculino; $F$ = feminino; $E F$ = Ensino Fundamental; $E M=$ Ensino Médio; SUP = Superior; PÓS = Pósgraduação. Cada categoria de escolaridade combinou o status completo e incompleto.

Table 2: Overall description of people interviewed in the Urban and Waterfall groups. Codes: $N=$ number of people; $\mathrm{M}=$ male; $\mathrm{F}$ = female; $\mathrm{EF}=$ elementary school; $\mathrm{EM}=$ high school; SUP = under-graduate course;

PÓS = graduate course. Each category combined the status "complete" and "incomplete".

\begin{tabular}{|c|c|c|c|c|c|c|c|c|c|c|}
\hline \multirow{2}{*}{ GRUPO } & \multirow{2}{*}{$\mathbf{N}$} & \multicolumn{2}{|c|}{ SEXO } & \multicolumn{3}{|c|}{ IDADE } & \multicolumn{4}{|c|}{ ESCOLARIDADE } \\
\hline & & $M$ & $\mathrm{~F}$ & MIN. & MÉD. & MÁX. & $\mathrm{EF}$ & EM & SUP & PÓS \\
\hline & 45 & 58 & 42 & 18 & 27 & 50 & 11 & 49 & 18 & 22 \\
\hline Cachoeira & 109 & 55 & 45 & 18 & 30 & 67 & 15 & 47 & 21 & 17 \\
\hline
\end{tabular}

\section{Uso de áreas naturais}

A maior parte dos entrevistados, de ambos os grupos, declarou que frequenta áreas naturais no máximo 3 vezes por ano (Tabela 3). A frequência de visitação da Cachoeira do Roncadeira é menor, considerando que a maior parte dos entrevistados declarou visitá-la menos de 2 vezes por ano.

Tabela 3: Frequência de visitação à Àreas Naturais e à Cachoeira do Roncadeira, Taquaruçu, TO, de acordo com os entrevistados do grupo Urbano e Cachoeira. Frequência = vezes/ano.

Table 3: Frequency use of Natural Areas and the Rocandeira Waterfall, Taquaruçu, TO, according to people interviewed in the Urban and Waterfall groups. Frequency $=$ times/year.

\begin{tabular}{lccccc}
\hline GRUPO & \multicolumn{5}{c}{ FREQUÊNCIA DE VISITAÇÃO (\%) } \\
& 1 vez & 2 vezes & 3 vezes & > 5 vezes & > 10 vezes \\
\hline Áreas Naturais & & & & & \\
Urbano & 44,2 & 23,3 & 14,0 & 9,3 & 9,3 \\
Cachoeira & 52,8 & 20,4 & 12,0 & 8,3 & 6,5 \\
Roncadeira & & & & & \\
Urbano & 31,8 & 11,4 & 20,5 & 18,2 & 18,2 \\
Cachoeira & 29,6 & 15,7 & 13,9 & 24,1 & 16,7 \\
\hline
\end{tabular}




\section{Percepção dos serviços ecossistêmicos}

De maneira geral, as pessoas entrevistadas apresentaram compreensão heterogênea sobre a existência/importância de serviços ecossistêmicos prestados pela área da Cachoeira da Roncadeira (Figura 2). Os entrevistados atribuíram importância Forte ou Média para muitos serviços, com destaque para biodiversidade, recursos hídricos, valor cultural, educação, regulação do clima e purificação da água. Por outro lado, muitos entrevistados atribuíram importância Fraca para alguns serviços, como produção de alimentos, matéria-prima, combustível e controle de doenças. Destaca-se também que houve atribuição Fraca ou Nula, por alguma parcela dos entrevistados, para quase todos os serviços.
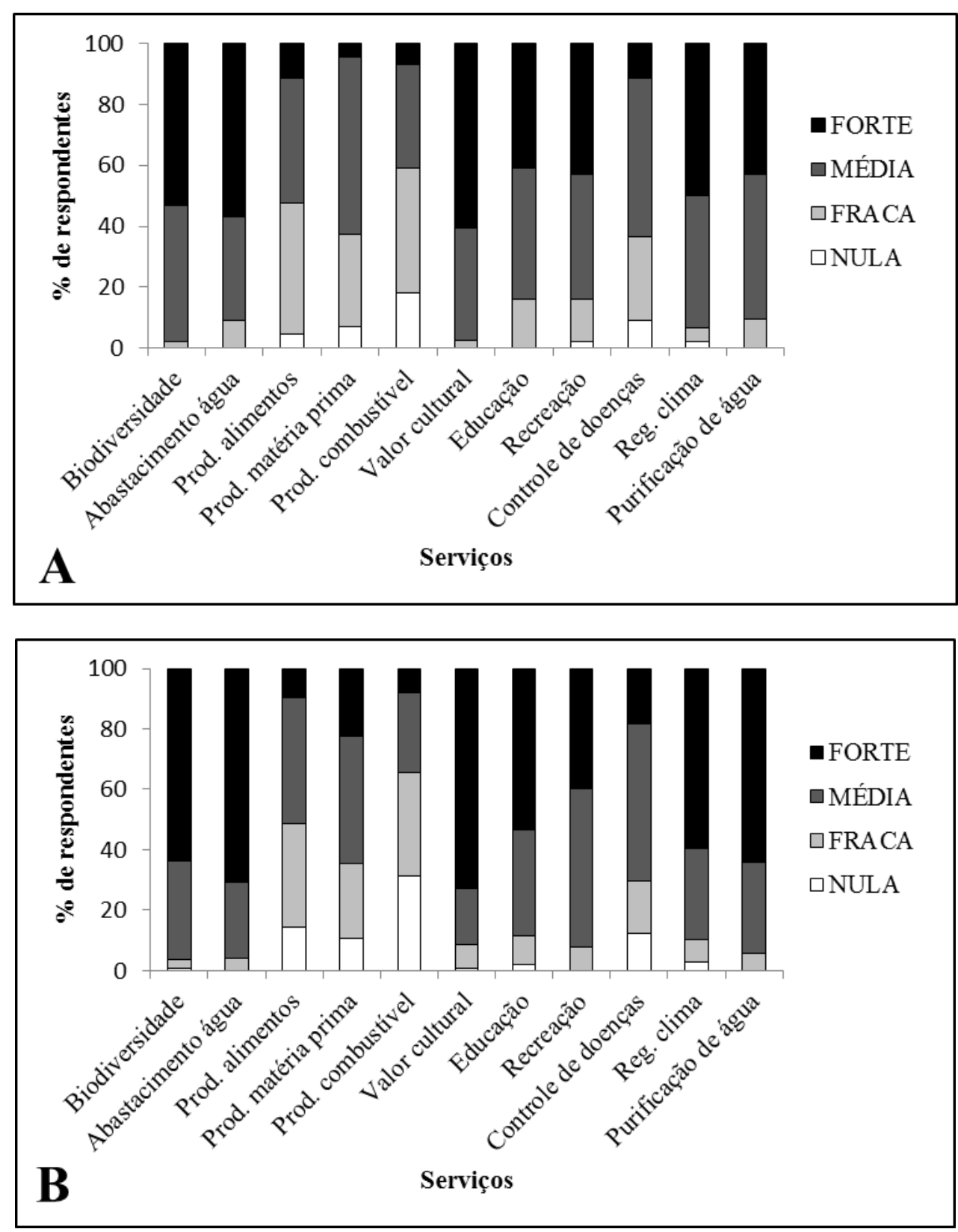

Figura 2: Importância da área para a provisão dos serviços ecossistêmicos indicados, de acordo com os entrevistados no grupo Urbano (A) e Cachoeira (B).

Figure 2: Importance of the area for the provision of ecosystem services, according to people interviewed in the Urban (A) and Waterfall (B) groups. 
Quase todos os entrevistados declararam sentir-se beneficiados por algum serviço ecossistêmico (Urbano $=84 \%$, Cachoeira $=96 \%$ ). No entanto, o percentual de beneficiados (declarados) foi baixo para a maior parte dos serviços (Figura 3A). $O$ padrão de respostas foi, no geral, semelhante entre os grupos, porém, o número de beneficiados no grupo Cachoeira foi maior em quase todos os serviços. Entre os serviços com maior reconhecimento de benefício estiveram biodiversidade, recreação e cultura, embora o percentual de beneficiados não tenha excedido $70 \%$. Em seguida aparecem abastecimento de água, purificação de água, regulação do clima e educação, onde o número de beneficiados declarados não excedeu $40 \%$. Os demais serviços tiveram pouca indicação como gerador de benefício. Agrupando os serviços em classes (Figura 3B), observamos que os benefícios gerados por serviços de Provisão e Cultural tendem a ser mais reconhecidos; menores percepções de benefícios estiveram atreladas aos serviços de Regulação.
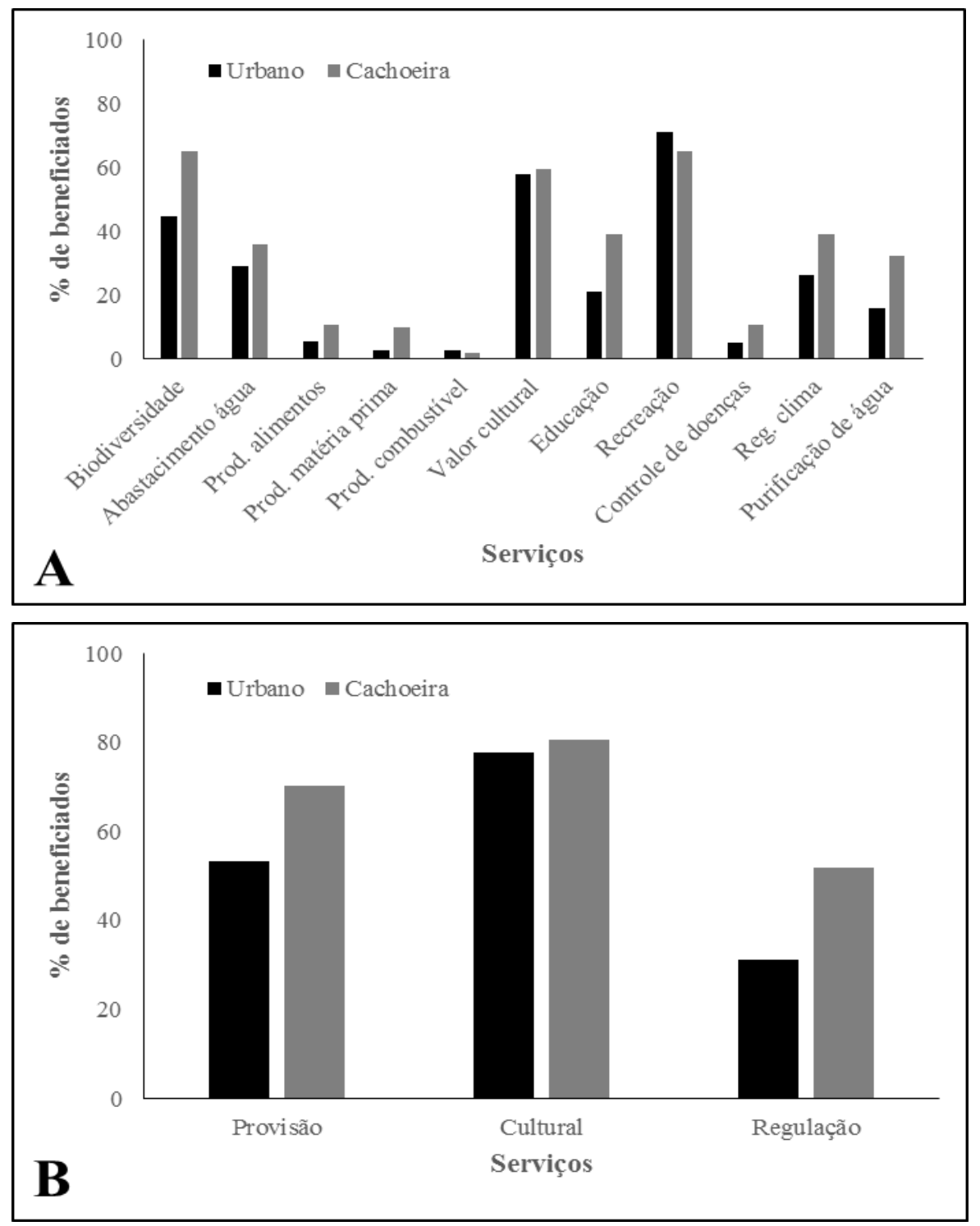

Figura 3: Percentual de entrevistados (grupos Urbano e Cachoeira) que declarou sentir-se beneficiado por cada serviço ecossistêmico (A), e agrupando-os nas categorias do Millennium Ecosystem Assessment (2005).

Figure 3: Percent of individuals (Urban and Waterfall groups) that felt benefited by each ecosystem service (A), and grouping these services according to the categories provided in Millennium Ecosystem Assessment (2005). 
Quando os entrevistados foram questionados sobre os cenários de desenvolvimento ou preservação da Cachoeira, aproximadamente metade dos entrevistados (Urbano $=47 \%$, Cachoeira $=60 \%$ ) se posicionou contra qualquer tipo de modificação na área (Figura 4). No caso, optaram por manter a área intocada sem que haja nenhuma transformação para a implantação de qualquer das atividades econômicas indicadas no questionário. Os demais respondentes demonstraram interesse na implantação de alguma atividade econômica na região, como pecuária, agricultura, hidrelétrica e urbanização. Quando questionados sobre o cenário de desenvolvimento econômico do Estado do Tocantins e a preservação de áreas naturais, mais de $80 \%$ dos entrevistados se declarou contra o desenvolvimento e perdas de áreas naturais, em ambos os grupos (Tabela 4).

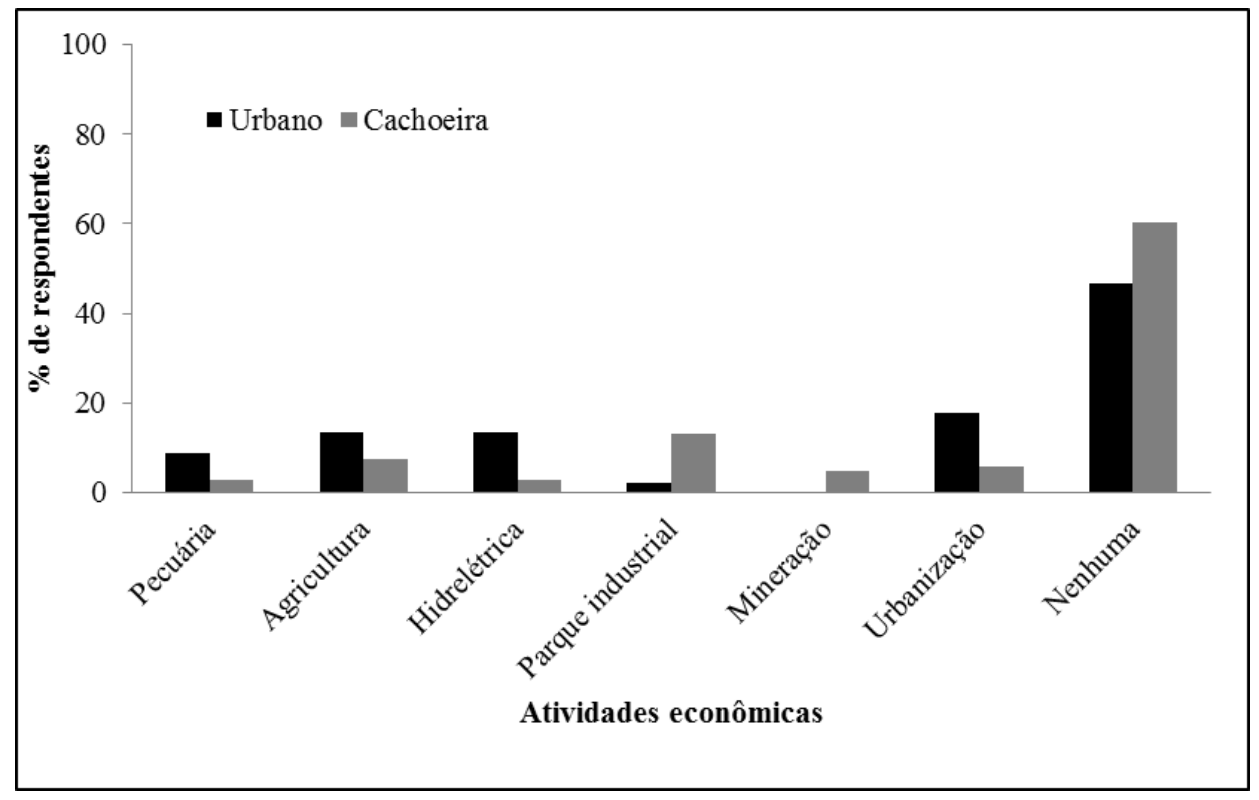

Figura 4: Percentual de entrevistados (grupos Urbano e Cachoeira) que declarou interesse no desenvolvimento de atividades econômicas na área da Cachoeira do Roncadeira, caso houvesse iniciativa de transformar a área em zona produção econômica.

Figure 4: Percent of individuals (Urban and Waterfall groups) that declared interest on the development of economic activities in the area of the Roncadeira Waterfall, if there was some initiative to convert the area into an economic zone.

Tabela 4: Percentual dos entrevistados que concordam (Sim), discordam (Não) ou concordam somente se houver elevado ganho econômico (Ganho), quanto à perda de áreas naturais para o desenvolvimento econômico do Estado do Tocantins.

Table 4: Percentage of individuals that agree (Sim), disagree (Não) or disagree only if there is economic profits (Ganho), with the loss of natural areas in face of economic development across the Tocantins State.

\begin{tabular}{lccc}
\hline Grupos & Sim & Não & Ganho \\
\hline Urbano & 8,9 & 82,2 & 8,9 \\
Cachoeira & 11,3 & 82,5 & 5,8 \\
\hline
\end{tabular}




\section{Discussão}

O presente trabalho investigou a percepção de pessoas sobre os serviços ecossistêmicos prestados pela cachoeira do córrego Roncadeira, Palmas, Tocantins, uma região de elevada importância turística. Nossos resultados indicam, de maneira geral, que as pessoas têm limitações na compreensão da importância do ambiente ripário como provedor de serviços ambientais essenciais, visto que a percepção foi heterogênea nos dois grupos investigados (Urbano e Cachoeira). Embora a maioria das pessoas tenha atribuído importância elevada para muitos dos serviços, houve percepção limitada por parte dos entrevistados para todos os serviços. Além disso, houve baixa compreensão dos benefícios derivados. Vale destacar que a pesquisa incidiu sobre pessoas que visitam com alguma frequência áreas naturais para fins de lazer e turismo, ou seja, que têm experiência direta com o ambiente natural. Além disso, os entrevistados apresentaram, no geral, elevado nível de instrução. Mesmo assim, houve entendimento limitado sobre a importância dos serviços e seus benefícios, indicando que pobre entendimento sobre o funcionamento e importância dos sistemas naturais.

Ao questionarmos a importância da área na provisão de serviços ecossistêmicos, constatamos que alguns serviços foram bem reconhecidos pelos entrevistados, como biodiversidade, recursos hídricos, recreação e cultura. O contato direto com esses serviços pode torná-los mais evidentes, pois os frequentadores usufruem deles ao longo da visitação. No entanto, nenhum serviço recebeu pontuação máxima por todos os entrevistados; além disso, todos os serviços receberam baixa pontuação (Nula ou Fraca) por algum respondente. Dentre os serviços pouco percebidos estiveram produção de alimentos (e.g. agricultura, frutos nativos), produção de matéria-prima (óleos, fibras, princípios ativos), produção de combustível (e.g. hidrelétrica) e controle de doenças (e.g. patógenos silvestres). Vale destacar que os serviços escolhidos e apresentados às pessoas foram aqueles típicos de ambientes ripários (BIGGS et al., 2017), ou seja, serviços comuns e inerentes a esse tipo de ecossistema. Informações prévias sobre sistemas ripários permitiria a identificação de tais serviços. A baixa percepção registrada, entretanto, não é incomum se considerarmos que os serviços são produtos de complexas interações biológicas, químicas e físicas (NAEEM et al., 1999; HOOPER et al., 2005); muitos deles não se apresentam claramente às pessoas (POSTEL; CARPENTER, 2007), visto que não são perceptíveis ao senso-comum. Essa falta de conhecimento sobre $o$ assunto não se limita ao leigo, pois as autoridades e gestores também se mostram muito desinformados (AZEVEDO-SANTOS et al., 2017). O cenário sugere a necessidade de se aprimorar a difusão de informações sobre o papel dos ecossistemas e a dependência das atividades humanas pelos serviços ambientais.

Todos os entrevistados declararam se sentir beneficiados por algum serviço. Os serviços que geraram maior percepção de benefício foram aqueles atrelados aos objetivos da visitação (i.e. turismo e recreação), no caso, biodiversidade, recreação e valor cultural. É notório que a procura por áreas naturais é motivada principalmente pelo desejo de entrar em contato com a natureza (MAMEDE et al., 2017), conforme relatado pelos próprios entrevistados (dados não apresentados), o que facilita a percepção de que o ambiente ripário é gerador de diversidade animal e vegetal, oportunidade de turismo/recreação e experiência cultural. Percebe-se, na verdade, que os entrevistados reconheceram apenas os benefícios gerados em escala local e imediata, sendo incapazes de estabelecer conexões causais em 
escalas espaciais maiores - caso dos sistemas fluviais, em que os componentes da bacia de drenagem aparecem interligados em múltiplas escalas, i.e. fenômenos locais provocam efeitos em escalas regionais, e vice-versa (GRANT et al., 2007; ABELL et al., 2011; McCLUNEY et al., 2014). Nisso, a percepção de benefício foi baixa para os demais serviços. Vale destacar que elencamos nessa pesquisa os serviços com efeitos diretos ou indiretos, em escala local e regional, nas atividades humanas conduzidas na região de Taquaruçu e Palmas. A deficiência de percepção é facilmente ilustrada pelo fato do ribeirão Taquaruçu ser o manancial hídrico que abastece a cidade de Palmas e região; no entanto, os respondentes não foram capazes de reconhecer a função do ribeirão na manutenção, produção e purificação de água potável. É curioso também o baixo reconhecimento dos benefícios gerados na regulação climática, uma vez que o assunto está em voga na mídia (i.e. aquecimento global), e a região do ribeirão Taquaruçu é coberta por florestas (i.e. ripária e Cerrado). A falta de informação de qualidade e contextualização, novamente, devem explicar esse quadro. Muitos trabalhos científicos têm mostrado a importância de áreas ripárias na geração de serviços ambientais essenciais e insubstituíveis (MILLENIUM ECOSYSTEM ASSESSMENT, 2005a; POSTEL; CARPENTER, 2007; BIGGS et al., 2017), principalmente aqueles atrelados a produção de água potável. Essa informação, obviamente, não tem sido veiculada de maneira adequada à população e autoridades, seja pela mídia, redes sociais ou ensino formal. Cabe destacar a crise hídrica que assolou as metrópoles do Sudeste, principalmente na cidade de São Paulo e região, um problema crescente e sem solução simples (LIMA JUNIOR et al., 2015). Nesse caso, o crescimento desordenado do ambiente urbano, a necessidade por desenvolvimento econômico, e a ampla remoção das florestas circundantes (Mata Atlântica), resultaram na destruição de mananciais e diminuição da recarga hídrica nos reservatórios. No caso da região de Taquaruçu, seu reconhecido valor turístico pode exercer papel positivo na manutenção das áreas naturais, porém o esclarecimento sobre a relevância dos demais serviços, alguns essenciais (i.e. geração de água), é necessário para o estabelecimento de políticas públicas que garantam a sustentabilidade das atividades econômicas na região.

Analisando cenários de desenvolvimento econômico na região, a maior parte dos entrevistados mostrou-se contrária a perda de áreas naturais em prol do desenvolvimento. Entretanto, parcela considerável de entrevistados se colocou favorável ao desenvolvimento de atividades econômicas na área da cachoeira do Roncadeira, como agricultura, pecuária, hidrelétricas e urbanização. Cabe destacar que 0 Estado do Tocantins, nas últimas décadas, experimentou rápido desenvolvimento nos setores de agronegócio e produção de energia hidrelétrica (COSTA et al., 2003; LIMA et al., 2016). Isso resultou na geração de recursos econômicos em curto prazo, porém com custos ambientais ainda não avaliados. A percepção de melhorias na geração de renda notoriamente exerce influência sobre as pessoas, o que deve explicar a concordância de muitos respondentes com a expansão de atividades produtivas em uma área natural de apelo turístico, mesmo que implique em degradação ambiental, ou mesmo a perda do potencial turístico. As transformações da paisagem podem indicar grosseiramente as mudanças ambientais em curso, mas não permitem inferir sobre a perda de serviços importantes, porém invisíveis, como a ciclagem de nutrientes, purificação da água, controle de doenças ou a biodiversidade de polinizadores. Nossos resultados sugerem, portanto, que mesmo as pessoas empáticas às áreas naturais, as quais criam vínculo e/ou se tornam usuários diretos do recurso, têm dificuldade em avaliar 
as consequências, em longo prazo, da perda de ecossistemas naturais e seus serviços.

O amplo declínio dos ecossistemas naturais precipita consequências negativas para as sociedades, incluindo a escassez do capital natural, elevação dos custos de serviços ambientais e risco de colapso de atividades produtivas (MILLENIUM ECOSYSTEM ASSESSMENT, 2005a; VOROSMARTY et al., 2010; CARDINALE et al., 2012). Nos últimos anos, as atividades humanas, para atender as crescentes demandas por alimentos, água e energia, têm afetado a capacidade dos ecossistemas em prover serviços essenciais (VITOUSEK et al., 1997), como manutenção da qualidade do ar e água, proteção contra catástrofes naturais, conservação e renovação do solo, provisão de fármacos e recursos genéticos. Algumas tentativas de quantificar o valor monetário desses bens e serviços demonstram valores extraordinariamente altos (WILSON; CARPENTER, 1999), superiores ao PIB mundial (COSTANZA et al. 1997). É imperativo, portanto, que as autoridades direcionem esforços para a conservação das áreas naturais remanescentes, muitas das quais, atualmente, tem elevado potencial turístico. A compreensão desse contexto pelo público e autoridades, entretanto, apresenta-se como um grande desafio. Programas de educação ambiental têm potencial de moldar o comportamento das pessoas, porém tais programas, seja nas escolas, projetos de extensão ou mídia, raramente abordam temas relativos à importância de se conservar sistemas ecológicos naturais. Nesse sentido, é imprescindível que a educação ambiental amplie seu escopo para além de ações específicas, de cunho local ou mesmo paliativo, de modo a abordar a relação entre os sistemas naturais e o bem-estar humano. Também relevante é a utilização de práticas in situ, como o ecoturismo, desde que aliem visitação com provisão de informações de qualidade (e.g. AZEREDO e ZAÚ, 2017; MAMEDE et al., 2017; LOUZEIRO et al., 2019), permitindo que o indivíduo vivencie experiências no campo, aprenda sobre 0 ecossistema, e reconheça seus serviços.

Concluindo, nosso trabalho revelou que a compreensão das pessoas sobre os serviços ambientais prestados por zonas ripárias é limitada; uma tendência que reflete, em grande medida, uma conduta em escala global. Nossos dados apontam a necessidade de esclarecimento para o público geral e autoridades sobre o funcionamento dos sistemas naturais, seus serviços e sua relação com o bem-estar humano.

\section{Referências}

ABELL, R. J; ALLAN, D.; LEHNER, B. Unlocking the potential of protected areas for freshwaters. Biological conservation, n.134, p.48-63, 2007.

AUERBACH, D.A.; DEISENROTH, D.B.; MCSHANE, R.R.; MCCLUNEY, K.E.; POFF, N.L. Beyond the concrete: Accounting for ecosystem services from freeflowing rivers. Ecosystem Services, v. 10, p. 1-5, 2014.

AZEREDO, T.A.; ZAÚ, A.S. Ecotourism and Environmental Education: connecting different concepts. Revista Brasileira de Ecoturismo, v.10, n.3, p.670-684, 2017.

AZEVEDO-SANTOS, V.M.; FEARNSIDE, P.M.; OLIVEIRA, C.S.; PADIAL, A.A.; PELICICE, F.M.; LIMA-JUNIOR, D.P.; SIMBERLOFF, D.; LOVEJOY, T.E.; MAGALHÃES, A.L.B.; ORSI, M.L.; AGOSTINHO, A.A.; ESTEVES, F.A.; POMPEU, P.S.; LAURANCE, W.F.; PETRERE JR., M.; MORMUL, R.P.; VITULE, J.R.S. 
Removing the abyss between conservation science and policy decision in Brazil. Biodiversity and Conservation, v.26, p.1745-1752, 2017.

BIGGS, J.; FUMETTI, S.V.; KELLY-QUINN, M. The importance of small waterbodies for biodiversity and ecosystem services: implications for policy makers. Hydrobiologia, v.793, p.3-39, 2017.

BURGER, J. R.; ALLEN, C. D.; BROWN, J. H.; BURNSIDE, W. R.; DAVIDSON, A. D.; FRISTOE, T. S.; HAMILTON, M. J.; MERCADO-SILVA, N.; NEKOLA, J.C.; OKIE, J.G.; ZUO, W. The macroecology of sustainability. PLoS Biology, v.10(6), e1001345, 2013.

CADOTTE, M.W.; CARSCADDEN, K.; MIROTCHNIK, N. Beyond species: functional diversity and the maintenance of ecological processes and services. Journal of Applied Ecology, v.48, p.1079-1087, 2011.

CARDINALE, B. J.; DUFFY, J. E.; GONZALEZ, A.; HOOPER, D.U.; PERRINGS, C.; VENAIL, P.; NARWANI, A.; MACE, G.M.; TILMAN, D.; WARDLE, D.A.; KINZIG, A.P.; DAILY, G.C.; LOREAU, M.; GRACE, J.B.; LARIGAUDERIE, A.; SRIVASTAVA, D.S.; NAEEM, S. Biodiversity loss and its impact on humanity. Nature, v.486, p.59-67, 2012.

CEBALLOS, G.; EHRLICH, P. R.; BARNOSKY, A. D.; GARCÍA, A.; PRINGLE, R. M.; PALMER, T. M. Accelerated modern human-induced species losses: Entering the sixth mass extinction. Science Advances, v.1, e1400253, 2015.

COSTA M.H.; BOTTA, A.; CARDILLE, J.A. Effects of largescale changes in land cover on the discharge of the Tocantins River, Southeastern Amazonia. Journal of Hydrology, v.283, p.206-217, 2003.

COSTANZA, R.; D'ARGE, R.; DE GROOT, R.S.; FARBER, S.; GRASSO, M.; HANNON, B.; LIMBURG, K.; NAEEM, S.; O'NEILL, R. V.; PARUELO, J.; RASKIN, R.G.; SUTTON, P.; VAN DEN BELT, M. The value of the world's ecosystem services and natural capital. Nature, v.387, p.253-260, 1997.

COSTANZA, R.; GROOT, R.; BRAAT, L.; KUBISZEWSKI, L.; FIORAMONTI L.; SUTTON, P.; FARBER, S.; GRASSO, M. Twenty years of ecosystem services: How far have we come and how far do we still need to go? Ecosystem Services v.28, p.1-16, 2017.

DAILY, G.C.; ALEXANDER, S.; EHRLICH, P.R.; GOULDER, L.; LUBCHENCO, J.; MATSON, P.A.; MOONEY, H.A.; POSTEL, S.; SCHNEIDER, S.H.; TILMAN, D.; WOODWELL, G.M. Ecosystem Services: Benefits Supplied to Human Societies by Natural Ecosystems. Ecological Society of America (Issues in Ecology), n.2, p.116, 1997.

GRANT, E.H.C.; LOWE, W.H.; FAGAN, W.F. Living in the branches: population dynamics and ecological processes in dendritic networks. Ecology Letters, v.10, p.165-175, 2007.

HOOPER, D.U.; CHAPIN III, F.S.; EWEL, J.J.; HECTOR, A.; INCHAUSTI, P.; LAVOREL, S.; LAWTON, J.H.; LODGE, D.M.; LOREAU, M.; NAEEM, S.; SCHMID, B.; SETALA, H.; SYMSTAD, A.J.; VANDERMEER, J.; WARDLE, D.A. Effects of biodiversity on ecosystem functioning: a consensus of current knowledge. Ecological Monographs, v.75(1), p. 3-35, 2005. 
LIMA, A.C.; AGOSTINHO, C.S.; SAYANDA, D.; PELICICE, F.M.; SOARES, A.M.V.M.; MONAGHAN K.A. The rise and fall of fish diversity in a Neotropical river after impoundment. Hydrobiologia, v.763, p.207-221, 2016.

LIMA JUNIOR, D.P.; MAGALHÃES, A.L.B.; VITULE, J.R.S. Dams, politics and drought threat: The march of folly in Brazilian freshwaters ecosystems. Natureza \& Conservacão, v.13, p.196-198, 2015.

LOUZEIRO, F.A.S. Experimentando o conhecimento: o Turismo Pedagógico como ferramenta para o Ensino Profissional. Revista Brasileira de Ecoturismo, v.12, n.1, p.55-66, 2019.

MAMEDE, S.; BENITES, M.; SABINO, J.; ALHO, C.J.R. Ecoturismo na região turística Caminho dos Ipês: conexões entre identidade biofílica e usufruto dos serviços ecossistêmicos. Revista Brasileira de Ecoturismo, v.10, n.4, p.938-957, 2017.

MCCLAIN, M. E.; COSSíO, R. E. The use of riparian environments in the rural Peruvian Amazon. Environmental Conservation. Environmental Conservation, v.3, n.30, p.242-248, 2003.

MCCLUNEY K.E.; POFF, N.L.; PALMER, M.A.; THORP, J.H.; POOLE, G.C.; WILLIAMS, B.S.; WILLIAMS, M.R.; BARON, J.S. Riverine macrosystems ecology: sensitivity, resistance, and resilience of whole river basins with human alterations. Frontiers in Ecology and the Environment, v.12, p.48-58, 2014.

MILLENNIUM ECOSYSTEM ASSESSMENT. Ecosystem and Human Well-Being. Washington DC: World Resources Institute, 2005a.100p.

MILLENNIUM ECOSYSTEM ASSESSMEN. Ecosystems and Human Well-being: Wetlands and Water Synthesis. Washington DC: World Resources Institute, 2005b. $80 p$.

NAEEM, S.; CHAPIN III, F. S.; COSTANZA, R.; EHRLICH, P. R.; GOLLEY, F. B.; HOOPER, D. U.; LAWTON, J. H.; O'NEILL, R. V.; MOONEY, H. A.; SALA, O. E.; SYMSTAD, A. J.; TILMAN, D. Biodiversity and ecosystem functioning: maintaining natural life support processes. Ecological Society of America (Issues in Ecology), n. 4, p.1-11, 1999.

NAIMAN, R.J.; DUDGEON, D. Global alteration of freshwaters: influences on human and environmental well-being. Ecological Restoration, v.26, p.865-873, 2011.

POSTEL, S.; CARPENTER, S. Freshwater Ecosystem Services. In: DAILY, G. C. (Eds). Nature's Services. 1 ed. Washington D.C.: Island Press, 1997. p.195-214.

TURNER, W.R.; BRANDON, K.; BROOKS, T.M.; COSTANZA, R.; FONSECA, R.A.B.; PORTELA, R. Global Conservation of Biodiversity and Ecosystem Services. BioScience, v. 57, n.10, p.868-873, 2007.

VITOUSEK, P. M.; MOONEY, H. A.; LUBCHENCO, J.; MELILLO, J. M. Human Domination of Earth's Ecosystems. Science, v.277, p.494-499, 1997.

VÖRÖSMARTY, C.J.; MCINTYRE, P.B.; GESSNER, M.O.; DUDGEON, D.; PRUSEVICH, A.; GREEN, P.; GLIDDEN, S.; BUNN, S.E.; SULLIVAN, C.A.; REIDY LIERMANN, C.; DAVIES, P.M. Global threats to human water security and river biodiversity. Nature, v.467, p. 555-561, 2010. 
WILSON, M.A.; CARPENTER, S.R. Economic Valuation of Freshwater Ecosystem Services in the United States: 1971-1997. Ecological Applications, v.9, n.3., p.772783, 1999.

\section{Agradecimentos}

Agradecemos à equipe da Cachoeira do Roncadeira pelo apoio logístico durante o trabalho de campo. Agradecemos também a todas as pessoas que gentilmente responderam ao questionário. Fernando $\mathrm{M}$. Pelicice recebeu bolsa de produtividade em pesquisa do CNPq.

Nibelle Aires Lira: Universidade Federal de Tocantins, Porto Nacional, TO, Brasil.

E-mail: nibelleaires@gmail.com

Link para o currículo Lattes: http://lattes.cnpq.br/9778286501522747

Fernando Mayer Pelicice: Universidade Federal de Tocantins, Porto Nacional, TO, Brasil.

E-mail: fmpelicice@gmail.com

Link para o currículo Lattes: http://lattes.cnpq.br/4838910413112753

Data de submissão: 08 de abril de 2019

Data de recebimento de correções: 24 de março de 2020

Data do aceite: 24 de março de 2020

Avaliado anonimamente 